\title{
Características físicas y composicionales de pastas de gres porcelánico con arcillas de Teruel
}

\author{
A. LAZARO ${ }^{1}$, C. GARCíA PORTILLO ${ }^{2}$, J. DE LA TORRE ${ }^{3}$, J. BASTIDA ${ }^{4}$
}

\begin{abstract}
Department of the Built Environment, Eindhoven University of Technology (Paises Bajos), ²Escuela Superior de Cerámica de Manises (España), ${ }^{3}$ Departamento de Ingeniería Química de la Universidad de Valencia (España) y ${ }^{4}$ Departamento de Geología de la Universidad de Valencia (España).
\end{abstract}

\begin{abstract}
Se desarrollaron pastas de gres porcelánico, elaboradas con arcillas de la zona minera de Teruel, con adiciones de feldespatos comerciales y sin adición de cuarzo, en el intervalo de temperatura 1120-1220. Se incluyen datos de absorción de agua, contracción lineal, resistencia a flexión y determinaciones de blancura $\left(\mathrm{L} \mathrm{a}{ }^{*} \mathrm{~b}^{*}\right.$ ), así como estimaciones semicuantitativas de fases cristalinas por el método de las intensidad de referencia. Se obtuvieron resultados cumpliendo con la norma ISO -13006 respecto a absorción de agua y resistencia mecánica a $1185^{\circ} \mathrm{C}$. Además, se demostró la conveniencia del método de las intensidades de referencia como alternativa a métodos cuantitativos más avanzados.
\end{abstract}

Palabras clave: Materias primas, gres porcelánico, ball clays, Teruel, análisis semicuantitativo.

Compositional and physical characteristics of porcelain tile bodies with clays from Teruel.

Stoneware pots were produced in the temperature range 1120-1220 using as raw materials ball clays from the mining area of Teruel (Spain) with additions of feldspars and without quartz additions. Data about water absorption, shrinkage, flexural strength and colour space $\left(\mathrm{L}^{*} \mathrm{~b}^{*}\right)$ are provided, as well as semiquantitative estimations of the crystalline phases in the bodies using the reference intensity method. The results fulfil the norm ISO-13006 regarding water absorption and mechanical strength at $1185^{\circ} \mathrm{C}$. In addition, it is shown that the intensity reference method was a good alternative to more advanced quantitative methods.

Key words: Raw materials, stoneware, ball clays, Teruel, semiquantitative analysis.

\section{INTRODUCCIÓN Y OBJETIVO.}

Las arcillas plásticas de cocción blanca o "ball clays" se destinan principalmente a pastas cerámicas para gres blanco, gres porcelánico y revestimientos de pasta blanca porosa y en menores cuantías para esmaltes, engobes y pastas de loza, porcelana y sanitarios. Las arcillas utilizadas en el sector cerámico nacional son principalmente de procedencia Española $(67 \%)$ aunque la cantidad importada (33\%) de Gran Bretaña, Alemania, Francia y Ucrania va en aumento. El consumo de ball clays en España alcanzó un volumen de 1.500 .000 t/ año, con un valor de 36 M€ según público el ICOG en $2006^{(1)}$.

La dosificación de ball clays habitual para gres esmaltado es de $30-70 \%$ de procedencia nacional y de 10-20\% de importación y para gres porcelánico no esmaltado de 35-50 $\%$ de importación y hasta un $25 \%$ de caolín ${ }^{(1)}$. Cuando no se precisa una gran blancura, se aumenta la aportación de arcilla nacional. Las arcillas ball clays son constituyentes importantes de las pastas de porcelana triaxial en una síntesis muy documentada ${ }^{(2)}$ con diversos tipos de aplicaciones ${ }^{(3)}$ y han sido objeto de trabajos recientes ${ }^{(4,5,6,7,8 \text { y 9). La principal }}$ producción nacional de ball clays se localiza en Teruel. En la Tabla I se recogen ejemplos típicos de ball clays nacionales y de importación ${ }^{(1)}$ con datos de composición química y propiedades físicas.

La creciente implantación en el mercado de los pavimentos de gres porcelánico, tanto esmaltado como no, así como el aumento de costes asociados a la importación de materias primas extranjeras, hace interesante la sustitución de materias extranjeras por materias nacionales cuando este cambio no

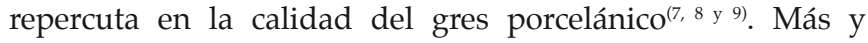
cuando, nos encontramos en estos momentos de grave crisis económica y de reestructuración del sector cerámico, en los cuales resulta imprescindible la disminución de costes manteniendo la calidad del producto final. De ahí que la utilización de materias primas de la zona para la producción de gres porcelánico con el fin de aumentar la competitividad del producto haya sido estudiada recientemente en diversos

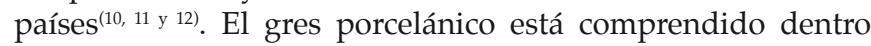
del grupo BIa (baldosas cerámicas prensadas en seco con absorción de agua $<0,5 \%$ y valor mínimo de resistencia a la flexión de $35 \mathrm{~N} / \mathrm{mm}^{2}$ ) de la norma ISO 13006 y UNE-EN 14411.

A nivel industrial, los parámetros de control del gres porcelánico que se analizan son las propiedades físicas 
TABLA I. COMPOSICIÓN QUÍMICA (\%) y PROPIEDADES TíPICAS DE BALL CLAYS UTILIZADOS PARA BALDOSAS.

\begin{tabular}{|c|c|c|c|c|c|c|}
\hline Arcilla & ${ }^{\text {aTE1 }}$ & TE2 & ${ }^{\mathrm{b}} \mathrm{U} 1$ & U2 & 'UK1 & UK2 \\
\hline $\mathrm{SiO}_{2}$ & 64.4 & 57.4 & 66.5 & 60.1 & 69.2 & 66.0 \\
\hline $\mathrm{Al}_{2} \mathrm{O}_{3}$ & 20.3 & 26.8 & 22.3 & 26.7 & 20.1 & 22.0 \\
\hline $\mathrm{Fe}_{2} \mathrm{O}_{3}$ & 2.3 & 2.4 & 0.9 & 0.9 & 0.8 & 0.9 \\
\hline $\mathrm{CaO}$ & 0.4 & 0.5 & 0.3 & 0.3 & 0.1 & 0.1 \\
\hline $\mathrm{MgO}$ & 0.3 & 0.5 & 0.5 & 0.6 & 0.3 & 0.3 \\
\hline $\mathrm{Na}_{2} \mathrm{O}$ & 0.2 & 0.3 & 0.4 & 0.5 & 0.3 & 0.3 \\
\hline $\mathrm{K}_{2} \mathrm{O}$ & 2.1 & 2.6 & 2.0 & 2.3 & 2.3 & 2.1 \\
\hline $\mathrm{TiO}_{2}$ & 1.0 & 1.0 & 1.4 & 1.4 & 1.6 & 1.5 \\
\hline PPC & 6.2 & 8.6 & 5.9 & 7.1 & 5.3 & 6.8 \\
\hline Rechazo $63 \mathrm{~mm}$ & 1.5 & 1.2 & 2.2 & 1.1 & 1.6 & 2.3 \\
\hline Contracción lineal $1120^{\circ} \mathrm{C}$ & 2.5 & 4.1 & 4.8 & 7.4 & 3.5 & 4.7 \\
\hline Contracción lineal $1220^{\circ} \mathrm{C}$ & 5.6 & 7.4 & 7.7 & 9.6 & 7 & 8.4 \\
\hline Absorción de agua $1120^{\circ} \mathrm{C}$ & 11.1 & 8.9 & 6.7 & 4.6 & 10.9 & 10.4 \\
\hline Absorción de agua $1220^{\circ} \mathrm{C}$ & 5.2 & 1.1 & 0.3 & 0.1 & 4.1 & 2.7 \\
\hline
\end{tabular}

${ }^{a} \mathrm{TE}$, hace referencia a arcillas de Teruel, ${ }^{b} \mathrm{U}$, hace referencia a arcillas de Ucrania, y cUK hace referencia a arcillas de Reino Unido.

TABLA II. COMPOSICIÓN QUÍMICA (\%) DE LAS MATERIAS PRIMAS EMPLEADAS.

\begin{tabular}{|c|c|c|c|c|c|c|}
\hline & AR1 & AR2 & $\mathrm{C} 1$ & $\mathrm{C} 2$ & FC & FS \\
\hline $\mathrm{SiO}_{2}$ & 65.04 & 60.56 & 80.00 & 57.06 & 74.13 & 76.18 \\
\hline $\mathrm{Al}_{2} \mathrm{O}_{3}$ & 21.07 & 24.55 & 12.33 & 27.34 & 14.76 & 16.05 \\
\hline $\mathrm{Fe}_{2} \mathrm{O}_{3}$ & 2.53 & 2.76 & 0.00 & 1.97 & 1.04 & 0.00 \\
\hline $\mathrm{TiO}_{2}$ & 0.92 & 1.01 & 0.23 & 0.40 & 0.48 & 0.52 \\
\hline $\mathrm{CaO}$ & 0.61 & 0.20 & 0.23 & 1.16 & 2.36 & 1.72 \\
\hline $\mathrm{MgO}$ & 0.41 & 0.66 & 0.84 & 0.39 & 3.14 & 0.16 \\
\hline $\mathrm{Na}_{2} \mathrm{O}$ & 0.15 & 0.19 & 0.05 & 0.09 & 3.71 & 5.10 \\
\hline $\mathrm{MnO}$ & 0.03 & 0.01 & 0.04 & 0.05 & 0.01 & 0.00 \\
\hline $\mathrm{P}_{2} \mathrm{O}_{5}$ & 0.10 & 0.07 & 0.06 & 0.08 & 0.14 & 0.10 \\
\hline PPC & 6.82 & 6.75 & 4.34 & 9.49 & 0.00 & 0.00 \\
\hline
\end{tabular}


(absorción de agua y resistencia a flexión o resistencia mecánica). En cambio a nivel de investigación, el análisis (semi)cuantitativo de las fases cristalinas y de la fase amorfa por el método de Rietveld ${ }^{(13)}$ es llevado a cabo frecuentemente junto con los ensayos físicos. El uso del método de Rietveld en los últimos años se ha hecho más frecuente debido al desarrollo y perfeccionamiento de los softwares que implementan dicho

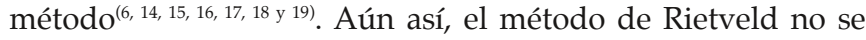
extiende al ámbito industrial, debido en gran medida, a la necesidad de experiencia en cristalografía del analista ${ }^{(18)}$. Un método similar, siendo más inexacto pero más sencillo de utilizar es el método de las intensidades de referencia. Este método es menos exacto que el método de Rietveld, pero nos permite seguir la evolución de las fases cristalinas y de la fase vítrea sin problemas, como queda demostrado en posteriores secciones.

En este trabajo se presenta la aplicación de ball clays de Teruel para la producción de gres porcelánico cumpliendo con la norma en cuanto a absorción de agua y resistencia mecánica. También se estudia la relación entre las propiedades físicas de las pastas cocidas y el contenido en mullita y fase vítrea de las mismas. Por último cabe destacar, que el análisis semicuantitativo de las fases cristalinas en las piezas fue realizado mediante el método de las intensidades de referencia implementado con el programa Eva del paquete Diffrac-Plus. Dicho método puede considerarse una buena alternativa a métodos cuantitativos más avanzados con mayor dificultad bien en la ejecución, utilizando mezclas de calibrado con patrón interno y/o adiciones ${ }^{(20)}$, o bien en el procesamiento de datos de difracción mediante análisis de Rietvield del registro

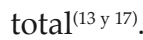

\section{MATERIALES Y MÉTODOS.}

Para la preparación de pastas se utilizaron arcillas (AR1 y AR2) y otros materiales (C1 y C2) procedentes de la zona minera de Teruel, además de feldespatos (FC y FS) procedentes de Cerdeña y Turquía. El análisis químico de las materias primas se presenta en la Tabla II. La distribución de tamaño de partícula de las materias primas fue analizada con el equipo Coulter LS 200. En la Tabla III se presentan los percentiles $d_{10^{\prime}} d_{50} \mathrm{y} \mathrm{d}_{90}$ de la distribución de tamaño de partícula de materias primas.

La elaboración de las pastas se realizó según la siguiente secuencia. Las materias primas se molieron por vía húmeda y por separado durante una hora en un molino de bolas de laboratorio. A continuación se secaron en estufa a $120{ }^{\circ} \mathrm{C}$
TABLA III. DisTRIBUCiÓN DEL TAMAÑO DE PARTÍCULA PARA LOS PERCENTILES 10, 50 Y 90 DE LAS DISTINTAS MATERIAS PRIMAS.

\begin{tabular}{|c|c|c|c|}
\cline { 2 - 4 } \multicolumn{1}{c|}{} & $\mathrm{d}_{10}(\mu \mathrm{m})$ & $\mathrm{d}_{50}(\mu \mathrm{m})$ & $\mathbf{d}_{90}(\mu \mathrm{m})$ \\
\hline AR1 & 2.00 & 8.56 & 64.24 \\
\hline AR2 & 1.94 & 6.85 & 31.18 \\
\hline C1 & 2.31 & 11.38 & 35.71 \\
\hline C2 & 2.06 & 8.57 & 38.88 \\
\hline FC & 2.61 & 14.4 & 50.73 \\
\hline FS & 2.89 & 18.8 & 121.68 \\
\hline
\end{tabular}

hasta alcanzar peso constante. La adecuada proporción entre materiales plásticos y desgrasantes necesaria para conseguir una buena compactación en el conformado de las probetas, así como la adecuada proporción entre materiales refractarios y fundentes ${ }^{(21}$ y ${ }^{22)}$ nos lleva a preparar las composiciones indicadas en la tabla IV. Estas composiciones se muelen de nuevo durante 20 minutos para asegurar la mezcla íntima entre todos los componentes. A continuación se conformaron las probetas por prensado a $300 \mathrm{Kg} / \mathrm{cm}^{2}$ y $5,5 \%$ de humedad. Dichas probetas se cocieron en horno eléctrico en ciclos de 61 minutos, con 10 minutos de permanencia a la temperatura máxima; la curva de cocción se muestra en la Figura 1.

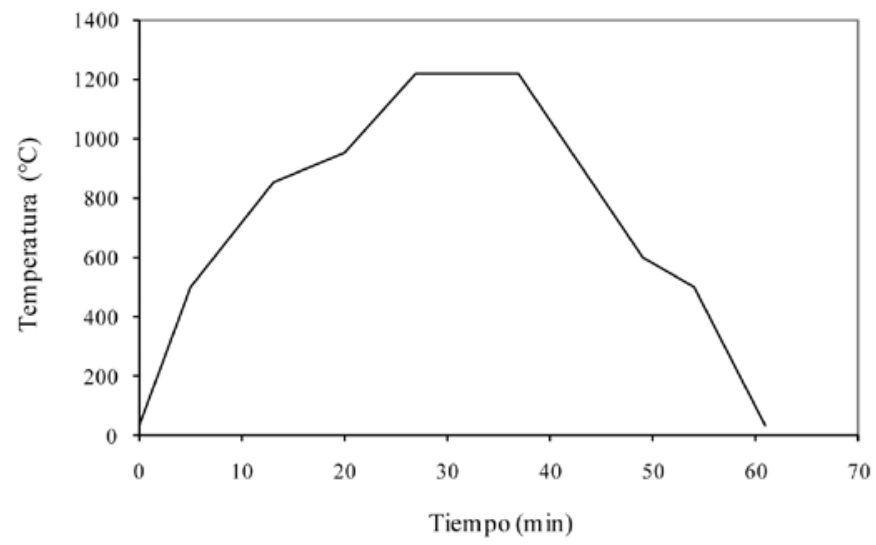

Figura 1. Curva de cocción.

TABLA IV. COMPOSICIÓN (\%) DE LAS PASTAS CERÁMICAS ENSAYADAS.

\begin{tabular}{|c|c|c|c|c|c|c|}
\hline MP/Pastas & A1 & A1+FC & A1+FS & A2 & A2+FC & A2+FS \\
\hline AR1 & 60.0 & 30.0 & 30.0 & 50.0 & 30.0 & 30.0 \\
\hline AR2 & 20.0 & 10.0 & 10.0 & 33.3 & 20.0 & 20.0 \\
\hline C1 & 20.0 & 10.0 & 10.0 & 8.3 & 5.0 & 5.0 \\
\hline C2 & & & & 8.3 & 40.0 & 5.0 \\
\hline FC & & 50.0 & & & & 40.0 \\
\hline FS & & & 50.0 & & & \\
\hline
\end{tabular}


La densidad aparente de las piezas prensadas fue determinada mediante el método de inmersión en mercurio, obteniéndose los valores que se indican en la tabla V. Dichos valores son muy cercanos a $1.95 \mathrm{~g} / \mathrm{cm}^{3}$, siendo este el valor industrial recomendado(23), confirmando así la bondad del proceso seguido. En la tabla $\mathrm{V}$ también se presentan los valores de resistencia mecánica de las probetas conformadas y secas. Dichos ensayos fueron realizados siguiendo la norma DIN 51030 con el Flexíometro Manneti FM 96.

Las probetas obtenidas fueron caracterizadas mediante fluorescencia de rayos $\mathrm{X}$ y mediante ensayos de absorción de agua, contracción lineal y resistencia a flexión. Se analizó la composición mineralógica por difracción de rayos $\mathrm{X}$ y se determinaron las coordenadas CIELAB $\left(\mathrm{L}^{*} \mathrm{a}^{*} \mathrm{~b}^{*}\right)$.

Los datos de composición química provienen de análisis realizados en los laboratorios Actlabs por fluorescencia de rayos X, utilizando la rutina WRA-XRA (4C). Para la caracterización por difracción de rayos $\mathrm{X}$ se utilizó un equipo Bruker D5005, según los procedimientos de preparación y de operación, descritos en un trabajo reciente sobre pastas de colaje $^{(24)}$. Para las determinaciones semicuantitativas de fases cristalinas se utilizó la versión del método de intensidades de referencia ${ }^{25}$ y 26$)$ implementada con el programa Eva del paquete Diffrac-Plus de Bruker, sin adición de patrón interno a las muestras. Como estimador de fase vítrea se da el área del difractograma excluyendo las áreas de los picos de las fases cristalinas, lo que supone una evaluación del área bajo el fondo general del registro.

Se han realizado observaciones en microscopia electrónica de barrido de emisión de campo (FESEM) con un equipo Hitachi 4100, trabajando a un voltaje de $30 \mathrm{KV}$ y a un potencial de extracción de $10 \mathrm{KV}$. Las muestras observadas son superficies de fractura de probetas cocidas, sometidas a ataque químico con vapores de ácido fluorhídrico durante 300 segundos de exposición, lavadas con agua destilada y secadas, y posteriormente metalizadas con un baño de oro en un metalizador modelo Struers Epovac, con tiempos de metalizado de un minuto.

Las coordenadas cromáticas CIELAB se midieron con un colorímetro Minolta CR-400/410, con lámpara de Xenon pulsante, sistema detector con 6 fotocélulas de silicio, operando con iluminación difusa sobre área circular de $22 \mathrm{~mm}$ de diámetro. Las coordenadas $L^{*}, a^{*}, b^{*}$ se calculan a partir de las coordenadas triestímulo X, Y, Z referidas al blanco patrón de la placa cerámica CR-A44.

\section{DISCUSIÓN Y RESULTADOS.}

\subsection{Análisis en crudo}

Las materias primas AR-1, AR-2 y C2 son ball-clays típicas de la zona de Teruel, presentado el material C2 mayor contenido en alúmina que las otras dos. El material $\mathrm{C} 1$ tiene un elevado contenido en $\mathrm{SiO}_{2}$ y bajo contenido en óxidos pigmentantes. La Figura 2 muestra los difractogramas de las materias primas utilizadas, en la cual se han representado las fichas de Cuarzo, Caolinita, Clinocloro y Albita.

En la Tabla VI se presentan los resultados del análisis químico de las distintas composiciones. En la tabla VII se presenta la composición química para pastas de referencia de gres porcelánico ${ }^{(23)}$. Los contenidos en sílice y en alúmina son similares en ambas tablas, mientras que el contenido en sodio es menor en nuestras pastas que en las pastas de referencia. Sin embargo este bajo contenido en sodio, se ve compensado con la presencia de otros óxidos fundentes. Así pues, la suma de los óxidos de $\mathrm{Ca}, \mathrm{Mg}, \mathrm{K}$ y $\mathrm{Na}$ resulta similar en nuestras pastas y en las pastas de referencia. La suma de óxido de hierro y titanio es mayor en nuestras pastas que en las pastas de referencia, lo que confiere a nuestras pastas cierta coloración (ver valores de coordenadas cromáticas CIELAB).

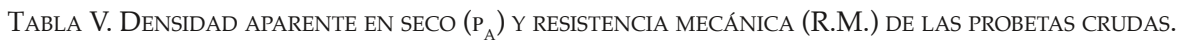

\begin{tabular}{|l|c|c|c|c|c|c|}
\cline { 2 - 7 } \multicolumn{1}{c|}{} & A1 & A1+FC & A1+FS & A2 & A2+FC & A2+FS \\
\hline$\rho_{\mathrm{A}}(\mathrm{g} / \mathrm{ml})$ & 1.991 & 1.888 & 1.879 & 1.966 & 1.914 & 1.917 \\
\hline R.M. $\left(\mathrm{N} / \mathrm{mm}^{2}\right)$ & 3.76 & 1.58 & 1.29 & 2.49 & 2.19 & 1.82 \\
\hline
\end{tabular}

TABLA VI. COMPOSICIÓN QUíMICA (\%) DE LAS PASTAS CERÁMICAS CRUDAS.

\begin{tabular}{|c|c|c|c|c|c|c|c|c|c|c|c|}
\cline { 2 - 11 } \multicolumn{1}{c|}{} & $\mathrm{SiO}_{2}$ & $\mathrm{Al}_{2} \mathrm{O}_{3}$ & $\mathrm{Fe}_{2} \mathrm{O}_{3}$ & $\mathrm{TiO}_{2}$ & $\mathrm{CaO}$ & $\mathrm{MgO}$ & $\mathrm{K}_{2} \mathrm{O}$ & $\mathrm{Na}_{2} \mathrm{O}$ & $\mathrm{MnO}$ & $\mathbf{P}_{2} \mathrm{O}_{5}$ & $\mathbf{P P C}$ \\
\hline $\mathrm{A} 1$ & 67.35 & 19.20 & 2.45 & 0.85 & 0.79 & 0.43 & 2.43 & 0.18 & 0.02 & 0.09 & 6.22 \\
\hline $\mathrm{A} 1+\mathrm{FC}$ & 70.83 & 16.78 & 1.42 & 0.69 & 1.87 & 2.02 & 1.43 & 1.61 & 0.11 & 0.12 & 3.11 \\
\hline $\mathrm{A} 1+\mathrm{FS}$ & 72.45 & 17.72 & 1.15 & 0.68 & 1.18 & 0.29 & 1.41 & 1.91 & 0.01 & 0.10 & 3.11 \\
\hline $\mathrm{A} 2$ & 63.85 & 21.86 & 2.89 & 0.89 & 0.47 & 0.50 & 2.59 & 0.17 & 0.02 & 0.09 & 6.68 \\
\hline $\mathrm{A} 2+\mathrm{FC}$ & 67.64 & 19.21 & 1.89 & 0.75 & 1.53 & 1.73 & 1.81 & 1.29 & 0.01 & 0.11 & 4.01 \\
\hline $\mathrm{A} 2+\mathrm{FS}$ & 68.41 & 20.08 & 1.63 & 0.74 & 1.24 & 0.44 & 1.79 & 1.56 & 0.01 & 0.09 & 4.01 \\
\hline
\end{tabular}


En la tabla V (donde se recogen los valores de densidad aparente y resistencia mecánica de las probetas crudas) se observan mayores valores de densidad aparente para la composición A1 que para la composición A2. En cambio estos valores se invierten en las composiciones que llevan feldespatos, pues las composiciones $\mathrm{A} 2+\mathrm{FC}$ y A2+FS presentan mayores valores de densidad aparente que las composiciones $\mathrm{A} 1+\mathrm{FC}$ y A1+FS. Esto se debe a que las composiciones 60/40 (material plástico/desgrasante) son más adecuadas que las composiciones 50/50 (material plástico/desgrasante), alcanzado mayores valores de densidad aparente. Una densidad aparente en crudo elevada nos permitirá obtener piezas con menor contracción en cocido, con menor deformación y con mayor intervalo de cocción, lo que es de suma importancia para conseguir piezas con buena estabilidad dimensional, ya que se trata de piezas de gres porcelánico producidas en ciclos de cocción rápidos. En cuanto a la relación entre resistencia mecánica en crudo y la densidad aparente, se observa que un incremento en la densidad aparente repercute en un aumento de la resistencia mecánica. Sin embargo, esta tendencia no se observa para las pastas con FS, pues mientras los valores de densidad aparente son similares para pastas con FC y FS, los valores de resistencia mecánica son mayores para las pastas con FC. Esto es debido a que el feldespato de Turquía tiene mayores tamaño de partícula, especialmente la fracción por encima del percentil 90.

\subsection{Análisis en cocido}

\subsubsection{PROPIEDADES FÍSICAS}

La Tabla VIII recoge los valores absorción de agua, contracción lineal y resistencia a flexión de las pastas cocidas a diferentes temperaturas. En dicha tabla se observa que la adición de feldespato disminuye la absorción de agua en todas las pastas y que dichas pastas cumplen la especificación fijada en la norma para el grupo Bla a partir de $1185{ }^{\circ} \mathrm{C}$. Dicha temperatura de cocción es relativamente baja para el gres porcelánico, repercutiendo en beneficios económicos y medioambientales para el proceso.
Curva de gresificación de A1 + FC

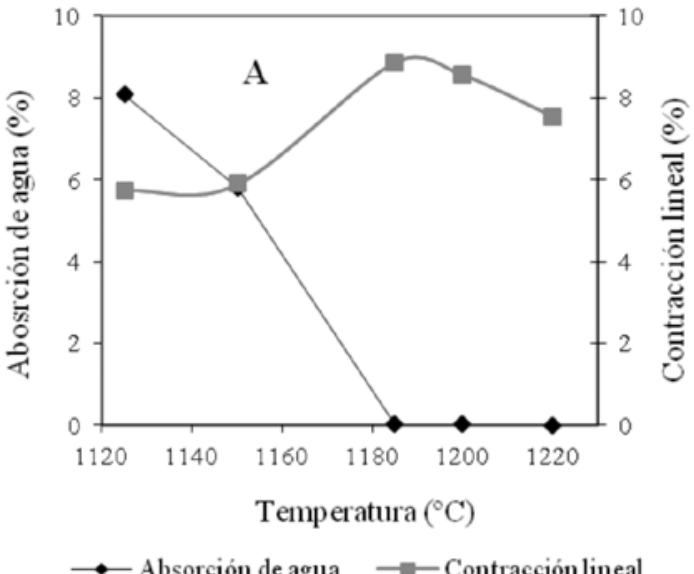

Curva de gresificación de $\mathrm{A} 2+\mathrm{FC}$

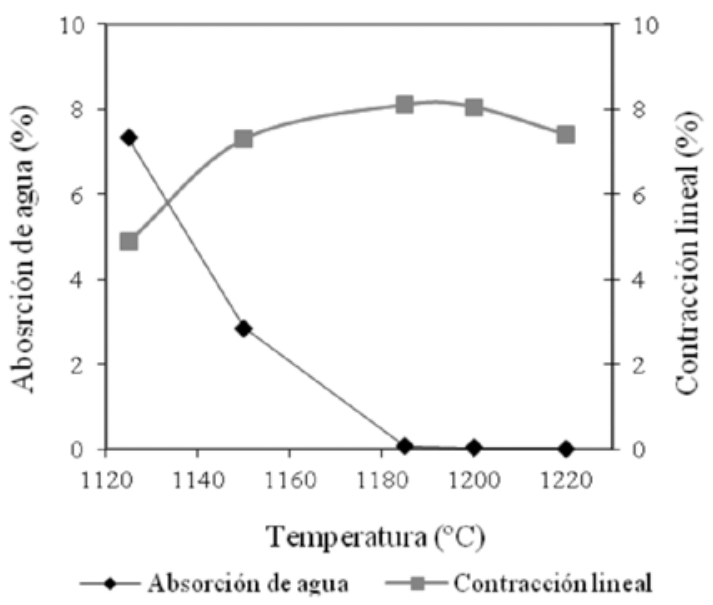

Figura 3. Diagrama de gresificación de la pasta A1+FC (A) y de la pasta $\mathrm{A} 2+\mathrm{FC}(\mathrm{B})$.

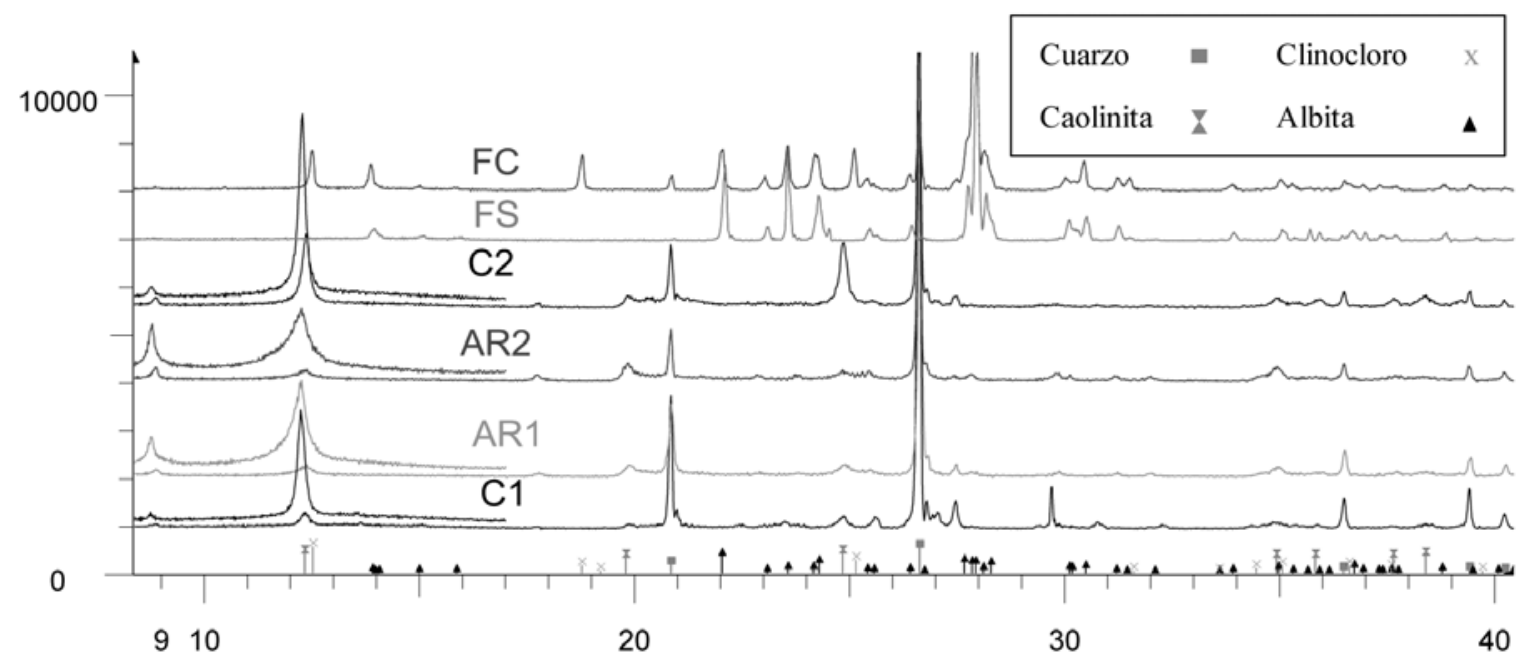

2-Theta - Scale

Figura 2. Difractogramas de las materias primas. 
TABLA VII. PASTA DE REFERENCIA PARA GRES PORCELÁNICO ${ }^{(23)}$.

\begin{tabular}{|l|c|c|c|c|c|c|c|}
\cline { 2 - 7 } \multicolumn{1}{c|}{} & $\mathrm{Al}_{2} \mathrm{O}_{3}$ & $\mathrm{SiO}_{2}$ & $\mathrm{Fe}_{2} \mathrm{O}_{3}+\mathrm{TiO}_{2}$ & $\mathrm{CaO}+\mathrm{MgO}$ & $\mathbf{K}_{2} \mathrm{O}$ & $\mathbf{N a}_{2} \mathrm{O}$ & 1.7 \\
\hline GP-1 & 21.0 & 67.0 & 0.8 & 0.8 & 4.5 & 4.2 \\
\hline GP-2 & 18.0 & 71.0 & 0.9 & 0.9 & 1.8 & 4.0 \\
\hline
\end{tabular}

TABLA VIII. VALORES DE ABSORCIÓN DE AGUA, CONTRACCIÓN LINEAL Y RESISTENCIA MECÁNICA PARA LAS DIFERENTES PASTAS A LAS DIFERENTES TEMPERATURAS.

\begin{tabular}{|c|c|c|c|c|c|c|}
\hline & Pastas & $1125\left({ }^{\circ} \mathrm{C}\right)$ & $1150\left({ }^{\circ} \mathrm{C}\right)$ & $1185\left({ }^{\circ} \mathrm{C}\right)$ & $1200\left({ }^{\circ} \mathrm{C}\right)$ & $1220\left({ }^{\circ} \mathrm{C}\right)$ \\
\hline 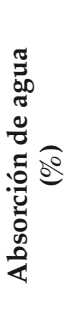 & $\begin{array}{c}\mathrm{A} 1 \\
\mathrm{~A} 2 \\
\mathrm{~A} 1+\mathrm{FC} \\
\mathrm{A} 1+\mathrm{FS} \\
\mathrm{A} 2+\mathrm{FC} \\
\mathrm{A} 2+\mathrm{FS}\end{array}$ & $\begin{array}{l}12.46 \\
10.53 \\
8.08 \\
11.09 \\
7.32 \\
9.82\end{array}$ & $\begin{array}{l}10.43 \\
8.70 \\
5.82 \\
7.19 \\
2.85 \\
4.76\end{array}$ & $\begin{array}{l}7.92 \\
7.71 \\
0.02 \\
0.18 \\
0.07 \\
0.25\end{array}$ & $\begin{array}{l}9.39 \\
6.64 \\
0.02 \\
0.01 \\
0.04 \\
0.02\end{array}$ & $\begin{array}{l}7.34 \\
5.26 \\
0.00 \\
0.01 \\
0.18 \\
0.00\end{array}$ \\
\hline 苂 & $\begin{array}{c}\mathrm{A} 1 \\
\mathrm{~A} 2 \\
\mathrm{~A} 1+\mathrm{FC} \\
\mathrm{A} 1+\mathrm{FS} \\
\mathrm{A} 2+\mathrm{FC} \\
\mathrm{A} 2+\mathrm{FS}\end{array}$ & $\begin{array}{l}1.60 \\
4.45 \\
5.75 \\
3.00 \\
4.90 \\
3.15\end{array}$ & $\begin{array}{l}2.60 \\
4.00 \\
5.90 \\
5.05 \\
7.30 \\
5.80\end{array}$ & $\begin{array}{l}3.60 \\
4.35 \\
8.85 \\
8.50 \\
8.10 \\
7.70\end{array}$ & $\begin{array}{l}3.45 \\
5.10 \\
8.55 \\
8.60 \\
8.05 \\
8.05\end{array}$ & $\begin{array}{l}4.25 \\
5.65 \\
7.55 \\
8.15 \\
7.40 \\
8.25\end{array}$ \\
\hline 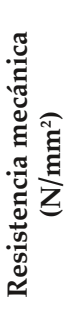 & $\begin{array}{c}\mathrm{A} 1 \\
\mathrm{~A} 2 \\
\mathrm{~A} 1+\mathrm{FC} \\
\mathrm{A} 1+\mathrm{FS} \\
\mathrm{A} 2+\mathrm{FC} \\
\mathrm{A} 2+\mathrm{FS}\end{array}$ & $\begin{array}{l}19.62 \\
39.24 \\
32.37 \\
23.54 \\
44.15 \\
30.41\end{array}$ & $\begin{array}{l}29.43 \\
36.49 \\
44.15 \\
34.34 \\
58.86 \\
49.05\end{array}$ & $\begin{array}{l}47.09 \\
39.24 \\
83.39 \\
58.86 \\
76.52 \\
68.67\end{array}$ & $\begin{array}{l}41.20 \\
43.65 \\
70.63 \\
76.52 \\
73.58 \\
80.44\end{array}$ & $\begin{array}{l}47.09 \\
40.61 \\
50.03 \\
72.59 \\
74.56 \\
83.39\end{array}$ \\
\hline
\end{tabular}

TABla IX. VALORES de las COORDENADAS CROMÁticas CIELAB L*, A* Y B*.

\begin{tabular}{|c|c|c|c|c|}
\hline Pastas & Temperatura $\left({ }^{\circ} \mathrm{C}\right)$ & $\mathbf{L}^{*}$ & $a^{*}$ & $\mathbf{b}^{*}$ \\
\hline A1 & 1185 & 73.13 & 6.40 & 15.67 \\
\hline A2 & 1185 & 73.01 & 7.00 & 16.08 \\
\hline $\mathrm{A} 1+\mathrm{FC}$ & 1185 & 57.98 & 3.46 & 10.62 \\
\hline $\mathrm{A} 1+\mathrm{FS}$ & 1185 & 59.11 & 4.41 & 10.89 \\
\hline $\mathrm{A} 2+\mathrm{FC}$ & 1185 & 58.35 & 3.51 & 10.90 \\
\hline $\mathrm{A} 2+\mathrm{FS}$ & 1185 & 61.28 & 5.12 & 12.32 \\
\hline A1 & 1200 & 73.93 & 6.39 & 15.78 \\
\hline A2 & 1200 & 71.33 & 6.21 & 15.23 \\
\hline $\mathrm{A} 1+\mathrm{FC}$ & 1200 & 57.24 & 2.87 & 10.29 \\
\hline $\mathrm{A} 1+\mathrm{FS}$ & 1200 & 55.82 & 3.77 & 9.72 \\
\hline $\mathrm{A} 2+\mathrm{FC}$ & 1200 & 57.20 & 2.92 & 10.33 \\
\hline $\mathrm{A} 2+\mathrm{FS}$ & 1200 & 56.08 & 3.57 & 9.76 \\
\hline
\end{tabular}


Con respecto a la resistencia a flexión se observa que mezclas con adición de feldespato y muestras de mayor temperatura de cocción presentan un mejor comportamiento. La adición de feldespato mejora la resistencia mecánica del producto acabado ya que cohesiona las fases cristalinas reduciendo la porosidad. A mayor temperatura de cocción de las pastas aumenta el contenido en mullita, y según Zoellner ${ }^{(27)}$ la resistencia de una porcelana depende únicamente del entramado de las agujas finas de mullita. Sin embargo esto no se cumple para el caso de la mezcla $\mathrm{A} 1+\mathrm{FC}$ en la cual la resistencia disminuye con la temperatura a partir de $1185^{\circ} \mathrm{C}$. Esto se debe a un aumento de la porosidad cerrada, como comentaremos a continuación.

De los resultados de la Tabla VIII, se identifican las pastas $\mathrm{A} 1+\mathrm{FC}$ y $\mathrm{A} 2+\mathrm{FC}$ a $1185{ }^{\circ} \mathrm{C}$ como idóneas para la producción de gres porcelánico a partir de dichas materias primas, ya que presentan una baja absorción de agua y una alta resistencia a flexión a una temperatura de cocción baja. A ello contribuye que el feldespato de Cerdeña (FC) posee un mayor contenido en óxidos fundentes y un menor contenido en alúmina que el feldespato de Turquía (FS), lo que unido al menor tamaño de partícula del FC, aporta una mayor reactividad a las pastas a las que se incorpora.

En la Figura 3 se muestran los diagramas de gresificación de dichas pastas a $1185{ }^{\circ} \mathrm{C}$. La pasta $\mathrm{A} 2+\mathrm{FC}$ presenta un intervalo de cocción más amplio, originado por su mayor contenido en $\mathrm{Al}_{2} \mathrm{O}_{3}$ (aportado por la materia prima C2) y menor contenido en feldespato, debido a la elección 60/40 (mineral arcilloso/feldespato). La pasta A1+FC (50/50, mineral arcilloso/feldespato) presenta una disminución de la contracción a partir de $1185^{\circ} \mathrm{C}$, lo que denota un aumento de la porosidad cerrada, dando lugar a su vez, a una menor resistencia mecánica.

En la Tabla IX se presentan los valores de las coordenadas cromáticas CIELAB L*, a* y b* para las temperaturas de cocción de 1185 y $1200{ }^{\circ} \mathrm{C}$. Dichos valores cromáticos corresponden a pastas claras de tonalidad beis-gris.
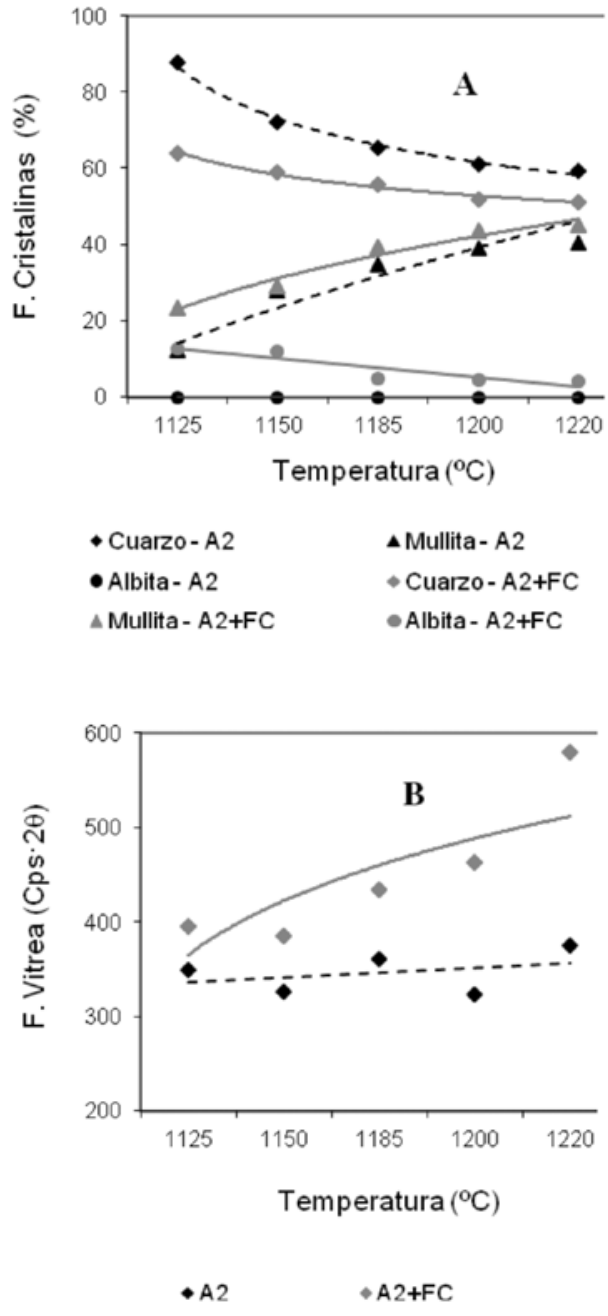

Figura 5. Gráficas de las fases cristalinas (A) y de la fase vítrea (B) con respecto a las temperaturas de cocción para la pasta $\mathrm{A} 2$ y para la pasta A2+FC.

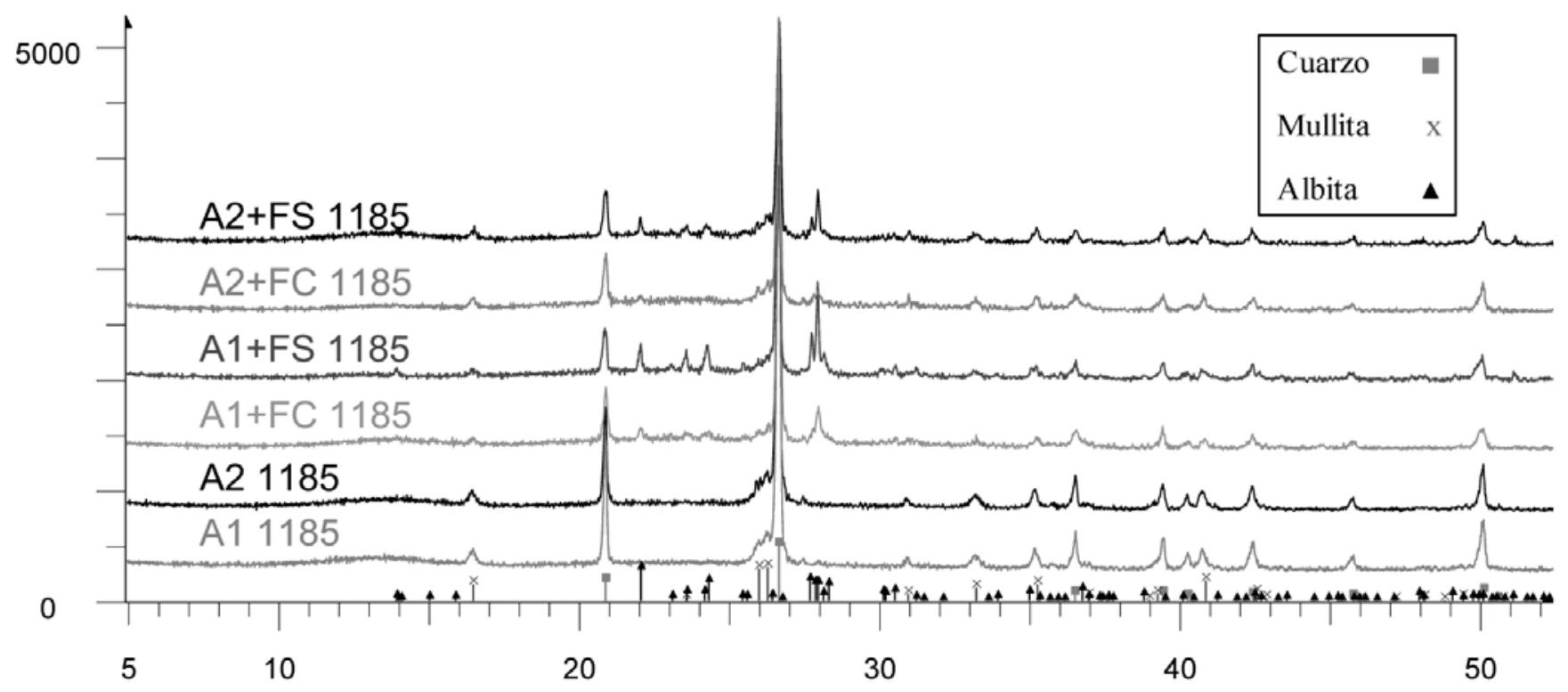

2-Theta - Scale

Figura 4. Difractogramas de las pastas cocidas a $1185^{\circ} \mathrm{C}$. 
TABLA X. DATOS DE FASES CRISTALINAS Y DE FASES VÍTREAS DE LAS PASTAS COCIDAS.

\begin{tabular}{|c|c|c|c|c|c|c|c|}
\hline & Temp. $\left({ }^{\circ} \mathrm{C}\right)$ & 1125 & 1150 & 1185 & 1200 & 1220 \\
\hline \multirow{18}{*}{ 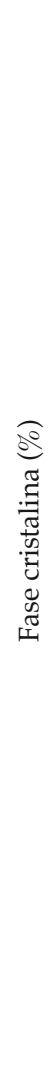 } & \multirow{3}{*}{ Z } & Cuarzo & 86.4 & 83.0 & 72.1 & 74.2 & 66.8 \\
\hline & & Mullita & 13.6 & 17.0 & 27.8 & 25.8 & 33.2 \\
\hline & & Albita & 0.0 & 0.0 & 0.0 & 0.0 & 0.0 \\
\hline & \multirow{3}{*}{ ये } & Cuarzo & 87.7 & 72.0 & 65.4 & 61.0 & 59.4 \\
\hline & & Mullita & 12.3 & 28.0 & 34.6 & 39.0 & 40.5 \\
\hline & & Albita & 0.0 & 0.0 & 0.0 & 0.0 & 0.0 \\
\hline & \multirow{3}{*}{$\begin{array}{l}U \\
\text { UT } \\
+ \\
+ \\
\end{array}$} & Cuarzo & 65.4 & 56.6 & 60.2 & 58.2 & 62.6 \\
\hline & & Mullita & 15.4 & 25.0 & 31.5 & 33.1 & 33.1 \\
\hline & & Albita & 19.1 & 18.4 & 8.2 & 8.7 & 4.3 \\
\hline & \multirow{3}{*}{$\begin{array}{l}\text { D } \\
\text { 岌 } \\
\underset{+}{\&}\end{array}$} & Cuarzo & 59.8 & 44.2 & 51.1 & 52.4 & 48.7 \\
\hline & & Mullita & 11.2 & 21.0 & 29.4 & 32.1 & 45.8 \\
\hline & & Albita & 29.0 & 34.8 & 19.5 & 15.4 & 5.5 \\
\hline & \multirow{3}{*}{ 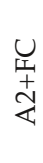 } & Cuarzo & 63.8 & 58.9 & 55.8 & 51.8 & 51.1 \\
\hline & & Mullita & 23.3 & 29.0 & 39.3 & 43.5 & 45.0 \\
\hline & & Albita & 12.8 & 12.1 & 4.9 & 4.6 & 4.0 \\
\hline & \multirow{3}{*}{$\begin{array}{l}\text { 足 } \\
+ \\
\stackrel{2}{4}\end{array}$} & Cuarzo & 60.7 & 55.6 & 50.7 & 52.7 & 56.1 \\
\hline & & Mullita & 16.6 & 24.4 & 37.3 & 37.0 & 37.0 \\
\hline & & Albita & 22.7 & 20.0 & 12.1 & 10.3 & 6.9 \\
\hline \multirow{6}{*}{\multicolumn{2}{|c|}{ 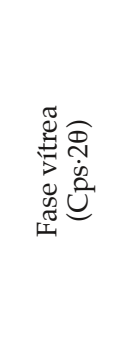 }} & A1 & 260.2 & 326.0 & 348.7 & 279.4 & 371.2 \\
\hline & & A2 & 349.1 & 325.4 & 360.2 & 323.5 & 375.4 \\
\hline & & $\mathrm{A} 1+\mathrm{FC}$ & 317.8 & 317.0 & 453.0 & 434.2 & 471.2 \\
\hline & & $\mathrm{A} 1+\mathrm{FS}$ & 263.1 & 309.1 & 390.1 & 431.0 & 466.9 \\
\hline & & $\mathrm{A} 2+\mathrm{FC}$ & 395.2 & 385.2 & 434.5 & 462.6 & 579.6 \\
\hline & & $\mathrm{A} 2+\mathrm{FS}$ & 308.6 & 358.0 & 453.0 & 451.5 & 485.6 \\
\hline
\end{tabular}

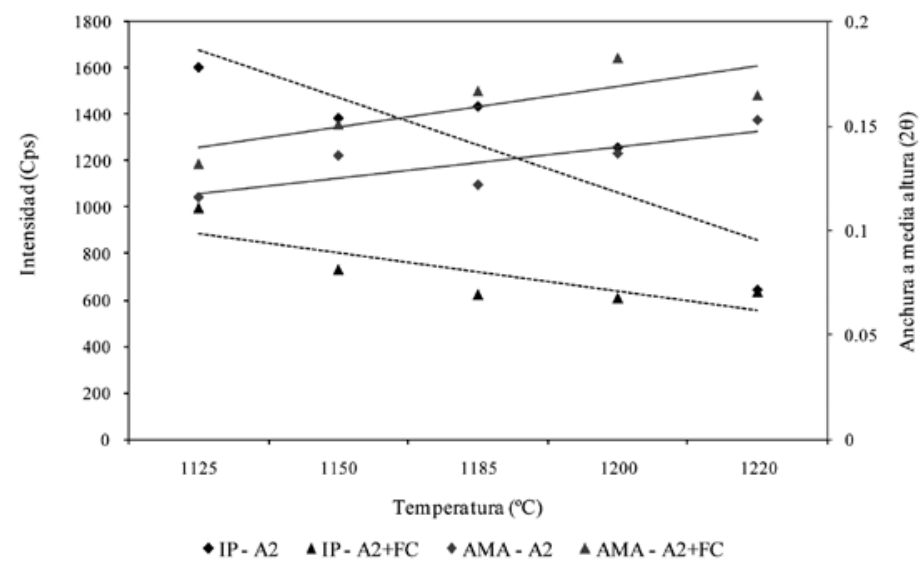

Figura 6. Intensidad de pico (IP) y anchura a media altura (AMA) de las pastas A2 y A2 + FC para el pico de cuarzo a d=3.34.

\subsubsection{FASES CRISTALINAS}

En la Figura 4 se muestran los difractogramas de las pastas cocidas a $1185{ }^{\circ} \mathrm{C}$, representado las fichas de Cuarzo, Mullita y Albita. La Tabla $X$ recoge los datos de las fases cristalinas y de las fases vítreas para todas las pastas. Los datos de las fases cristalinas son porcentajes del total de la fase cristalina y los datos relativos a la fase vítrea se dan en unidades arbitrarias. En la Figura 5 se representan estos valores para la pasta A2 y la pasta A2+FC. Dicha Figura permite visualizar la evolución de las fases cristalinas en la pasta A2 sin y con adición de feldespato, observándose una reducción del cuarzo y la albita y un aumento de la mullita y fase vítrea con la temperatura para la pasta A2 + FC. De la tabla $X$ cabe destacar los siguientes puntos: primero, que la pasta $\mathrm{A} 2$ + FC presenta un mayor contenido en mullita que la pasta $\mathrm{A} 1+\mathrm{FC}$ debido a su mayor contenido en $\mathrm{Al}_{2} \mathrm{O}_{3}$. Segundo, que la evolución de la fase vítrea con la temperatura es mucho más marcada para pastas con adición de feldespato. $\mathrm{Y}$ tercero, que las pastas con FS tienen mayor contenido de albita a $1185^{\circ} \mathrm{C}$ que las mismas pastas con FC, lo cual se debe a la mayor reactividad del feldespato de Cerdeña. El mayor contenido de albita para las composiciones con FS a $1185^{\circ} \mathrm{C}$ está en concordancia con los mayores valores de absorción de agua y menores valores de resistencia mecánica para dicha pasta. Por consiguiente, la evolución de las fases cristalinas y de la fase vítrea corresponde a la evolución esperada, lo cual pone de manifiesto la utilidad del método de las intensidades de referencia para la estimación semicuantitativa de fases cristalinas en pastas cerámicas, siempre y cuando se tengan valores adecuados de intensidades.

En la Figura 6 se representan los valores de intensidad de pico y anchura a media altura para el pico de cuarzo a $\mathrm{d}=3.34 \AA$ en las pastas A2 y A2 + FC. Puede apreciarse, que al aumentar la temperatura la cantidad de cuarzo disminuye y se reduce el tamaño de cristalito del cuarzo. Dicho fenómeno no es habitual en las pastas triaxiales de gres porcelánico y puede deberse a que en nuestro caso no se añade cuarzo como materia prima sino que las materias primas son ricas en cuarzo, especialmente la materia prima C1. Así el cuarzo presente es de menor tamaño de partícula y se encuentra íntimamente mezclado con otros minerales, lo que facilita la incorporación del cuarzo a la fase vítrea a las temperaturas de cocción ensayadas ${ }^{(28)}$.

En las Tablas XI y XII se recogen los valores de fase mullita, fase vítrea (F. V.), absorción de agua (A. A.) y resistencia mecánica (R. M.) para la pastas A1+FC y A2 +FC. Se observa que la absorción de agua disminuye con el aumento de la temperatura al contrario que la fase vítrea que aumenta. El contenido en mullita aumenta con la temperatura, al igual que la resistencia mecánica. En cambio si la fase vítrea no es tan abundante, como ocurre en las composiciones A1 y A2 sin adición de feldespato, el contenido en mullita no aumenta la resistencia mecánica tan efectivamente. Esto se debe a que las agujas de mullita no están cohesionadas por la fase vítrea ${ }^{(27)}$. Otro caso en el que el aumento del contenido en mullita no supone un aumento directo de la resistencia mecánica es cuando se produce un aumento de la porosidad cerrada, como ocurre en la pasta $\mathrm{A} 1+\mathrm{FC}$ a partir de $1185^{\circ} \mathrm{C}$.

En la Figura 7 se presentan fotos de FESEM de las composiciones sin adición de feldespato A1 y A2 cocidas a $1220^{\circ} \mathrm{C}$. Fueron seleccionadas las composiciones sin adición de 
feldespato por disponer de una menor cantidad de fase vítrea, lo que permite una mejor visualización de mullita y fueron seleccionadas a $1220{ }^{\circ} \mathrm{C}$ dado que el contenido en mullita es mayor a temperaturas elevadas. Las microfotografías realizadas muestran que la pasta A2 presenta una mayor cantidad de cristales pero de menor tamaño que la pasta A1, en concordancia con los resultados de las fases cristalinas (Tabla X) y con los valores del contenido en $\mathrm{Al}_{2} \mathrm{O}_{3}$. La buena concordancia entre el contenido de mullita y las fotografías de FESEM muestra nuevamente la utilidad del método de las intensidades de referencia para estimaciones semicuantitativas de fases cristalinas sin tener que recurrir a métodos más avanzados y laboriosos de análisis cuantitativo. Así, el método de las intensidades de referencia puede ser especialmente útil para la determinación semicuantitativa de las fases cristalinas para la industria, revelándose como un método ágil y simple que proporciona información adicional a los ensayos físicos.

La Tabla XIII recoge los datos de absorción de agua, contracción lineal y resistencia a flexión de las pastas escogidas a la temperatura seleccionada y los requerimientos para baldosas cerámicas grupo BIa (gres porcelánico) de las Normas ISO 13006 y UNE-EN 14411. De esta tabla se desprende que dichas pastas cumplen sobradamente con la norma a la temperatura de $1185^{\circ} \mathrm{C}$.

\section{CONCLUSIONES.}

Hay que resaltar pues, que formulando composiciones binarias con materias primas de Teruel y feldespato de Cerdeña, se obtienen pastas de gres porcelánico de tonalidad beis-gris con arreglo a especificaciones de la norma. Así, se alcanzan los requerimientos para la absorción de agua y para la resistencia a flexión a temperaturas de cocción relativamente bajas, suponiendo una ventaja competitiva con respecto a otras composiciones. El contenido elevado en $\mathrm{SiO}_{2}$ de las distintas materias primas posibilita la no utilización de cuarzo, permitiendo una buena adaptación de estas pastas a ciclos de cocción rápidos. El comportamiento apreciado es susceptible de ser mejorado mediante dosificación de arcillas más illíticas de la zona y/o de arcillas presentando caolinita y/o illita de menores tamaños de cristalito ${ }^{(29)}$.

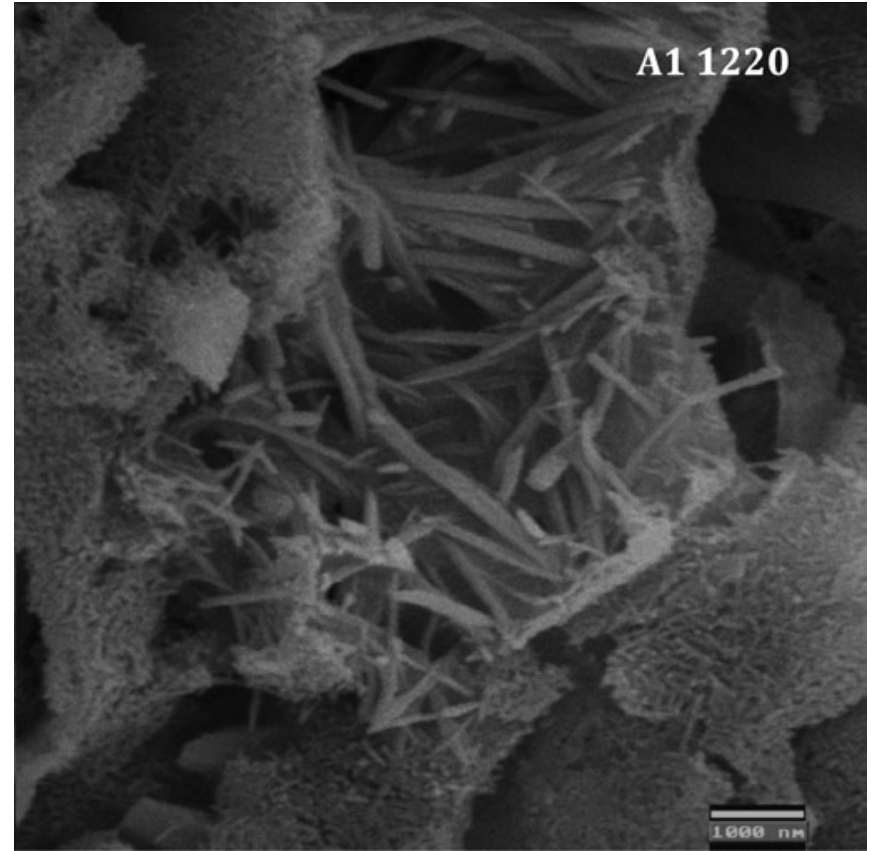

Figura 7. Fotos de FESEM de la superficie de las pastas A1 y A2 a $1220^{\circ} \mathrm{C}$ (escala gráfica $1000 \mathrm{~nm}$ ).

Tabla XIII. Propiedades de las pastas A1+FC y A2+FC a $1185^{\circ} \mathrm{C}$ y ESPECIFICACIONES DE LA NORMA IS0-13006.

\begin{tabular}{|c|c|c|c|}
\cline { 2 - 4 } \multicolumn{1}{c|}{} & A.A. (\%) & C.L. $(\%)$ & R.M. $\left(\mathbf{N} / \mathbf{m m}^{\mathbf{2}}\right)$ \\
\hline A1+FC & 0.02 & 8.85 & 83.39 \\
\hline A2+FC & 0.07 & 8.1 & 76.52 \\
\hline NORMA & $<0,5$ & - & $>35$ \\
\hline
\end{tabular}

TABla XI. DATOS DE CONTENIDO EN MUllita, FASE VÍtREA, ABSORCión DE AGUA Y RESISTENCIA MECÁNICA PARA LA PASTA A1+FC.

\begin{tabular}{|c|c|c|c|c|c|}
\cline { 2 - 6 } \multicolumn{1}{c|}{} & $\mathbf{1 1 2 5}\left({ }^{\circ} \mathbf{C}\right)$ & $\mathbf{1 1 5 0}\left({ }^{\circ} \mathbf{C}\right)$ & $\mathbf{1 1 8 5}\left({ }^{\circ} \mathbf{C}\right)$ & $\mathbf{1 2 0 0}\left({ }^{\circ} \mathbf{C}\right)$ & $\mathbf{1 2 2 0}\left({ }^{\circ} \mathbf{C}\right)$ \\
\hline Mullita (\%) & 15.4 & 25.0 & 31.5 & 33.1 & 33.1 \\
\hline F. V. $(\mathrm{Cps} \cdot 2 \theta)$ & 317.8 & 317.0 & 453.0 & 434.2 & 471.2 \\
\hline A. A. $(\%)$ & 8.08 & 5.82 & 0.02 & 0.02 & 0.00 \\
\hline R. M. $(\%)$ & 32.37 & 44.15 & 83.39 & 70.63 & 50.03 \\
\hline
\end{tabular}

TABla XII. DATOS DE CONTENIDO EN MULlitA, FASE VÍTREA, ABSORCIÓN DE AGUA Y RESISTENCIA MECÁNICA PARA LA PASTA A2+FC.

\begin{tabular}{|c|c|c|c|c|c|}
\cline { 2 - 6 } \multicolumn{1}{c|}{} & $\mathbf{1 1 2 5}\left({ }^{\circ} \mathbf{C}\right)$ & $\mathbf{1 1 5 0}\left({ }^{\circ} \mathbf{C}\right)$ & $\mathbf{1 1 8 5}\left({ }^{\circ} \mathbf{C}\right)$ & $\mathbf{1 2 0 0}\left({ }^{\circ} \mathbf{C}\right)$ & $\mathbf{1 2 2 0}\left({ }^{\circ} \mathbf{C}\right)$ \\
\hline Mullita $(\%)$ & 23.3 & 29.0 & 39.3 & 43.5 & 45.0 \\
\hline F. V. $(\mathrm{Cps} \cdot 2 \theta)$ & 395.2 & 385.2 & 434.5 & 462.6 & 579.6 \\
\hline A. A. $(\%)$ & 7.32 & 2.85 & 0.07 & 0.04 & 0.18 \\
\hline R. M. $(\%)$ & 44.15 & 58.86 & 76.52 & 73.58 & 74.56 \\
\hline
\end{tabular}


Otro aspecto que cabe resaltar es la concordancia entre los valores de las fases cristalinas con los datos de las propiedades físicas de las piezas (absorción de agua y resistencia mecánica) y con la composición química de las pastas cerámicas crudas. Lo cual pone de manifiesto la utilidad del método de las intensidades de referencia para el análisis semicuantitativo de las fases cristalinas como alternativa simple y rápida a métodos cuantitativos más avanzados.

\section{BIBLIOGRAFÍA.}

(1) ICOG. El sector de las arcillas en la provincia de Teruel. Gobierno de Aragón. Departamento de Industria, Comercio y Turismo. Servicio de Ordenación Minera. 159 pp, 2006.

(2) Carty, W. M., Senepati, U. Porcelain - Raw Materials, processing, Phase Evolution and Mechanical Behaviour. J. Amer. Ceram. Soc., 81, 3-20,1998.

(3) Norton, F. H. Cerámica Fina: Tecnología y aplicaciones. Omega, 504 pp., Barcelona, 1988.

(4) García Portillo, C., Milian, V., Lazaro, A., De la Torre, J., Bastida, J. Poster: Formulaciones de Gres Porcelánico con materias primas arcillosas de procedencia nacional. Qualicer 08, World forum on ceramic tile, 39-42, 2008.

(5) Carbajal, L., Rubio-Marcos, F., Bengochea, M. A., Fernandez, J. F. Properties related phase evolution in porcelain ceramics. J. European Ceram. Soc., 27, 13-15, 4065-4069, 2007.

(6) Ferrari, S., Gualtieri, A. F. The use of illitic clays in the production of stoneware tile ceramics. App. Clay Sci., 32, 73-81, 2006.

(7) Sánchez-Muñoz, L., Nebot-Díaz, I., Carda, J., Tuduri, B., Gracia, A., Cerisuelo, E. Obtención de soportes cerámicos nacionales de baja porosidad a partir de materia primas nacionales. Cerámica. Información, 27 (272), 3, 48-54, 2001.

(8) Sánchez, E., García-Ten, J., Quereda, P., Beltrán, V. Estudio de composiciones de gres rojo de muy baja porosidad. Técnica cerámica, 305, 902-909, 2002.

(9) Notari, M. D., Llop, J., Nebot, I., Barrachina, E., Ferrer, S., Carda, J. B. Estudio y caracterización de materias primas para la formulación de pastas cerámicas de gres porcelánico. Cerámica información, 34, 15, 45-61, 2008.

(10) Dondi, M., Iglesias, C., Dominguez, E., Guarini, G., Raimondo, M. The effect of kaolin properties in their behaviour in ceramic processing as illustrated by a range of kaolins from the Santa cruz and Chubut Provinces, Patagonia (Argentina). Applied clay science, 40, 143-158, 2007.

(11) Olupot, P. W., Jonsson, S., and Turan, S. Study of glazes and their effects on properties of triaxal electrical porcelains from Ugandan Minerals. Journal of Materials engineering and performance, 19, 1133-1142, 2009.

(12) Galos, K. Composition and ceramic properties of ball clays for porcelain stoneware tiles manufacture in Poland, Applied clay science, 51, 74-85, 2011.
(13) Rietveld, H.M. A profile refinement method for nuclear and magnetic structures. J. Appl. Crystallogr., 2, 65-71, 1969.

(14) Matteucci, F., Dondi, M., Guarini, G. Effect of soda-lime glass on sintering and technological properties of porcelain stoneware tiles. Ceram. Int., 28, 873-80, 2002.

(15) Tucci, A., Esposito, L., Malmusi, L., Rambaldi, E. New body mixes for porcelain stoneware tiles with improved mechanical characteristics. J. European Ceram. Soc., 27, 1875-81, 2007.

(16) Zanelli, C., Baldi, G., Dondi, M., Ercolani, G., Guarini, G., Raimondo, M. Glass-ceramic frits for porcelain stoneware bodies: effects on sintering, phase composition and technological properties. Ceram Int., 34, 455-65, 2008.

(17) Martin-Marquez, J., De la Torre, A.G., Aranda, M. A. G., Rincon, J. M. Romero, M. Evolution with temperature of crystalline and amorphous phases in porcelain stoneware. J. Amer. Ceram. Soc., 92, 229-234, 2009.

(18) Mahieux, P.Y., Aubert, J.E, Cyr, M., Coutand, M., Husson, B. Quantitative mineralogical composition of complex mineral wastes - Contribution of the Rietveld method. Waste Management, 30, 378-388, 2010.

(19) Gualteri, M.L., Romagnoli, M., Gualteri A.F. Influence of body composition on the technological properties and mineralogy of stoneware: A DOE and mineralogical-microstructural study. J. European Ceram., 31, 673-685, 2011.

(20) Monshi, A., and Messer, P. F. Double dilution X-ray diffraction method for quantitative phase analysis of multiple systems. British Ceramic Proceeding, 42, 201-212, 1989.

(21) Escardino A., Amorós J. L., Negre, F. C. Defectos de planariedad en las piezas de pavimento gresificado motivados por deformación piroplástica. Influencia de las variables del proceso. Taulells, 3. 1985.

(22) Garcia Portillo C., Lázaro V., Lazaro A. Reología de materiales cerámicos. Sevilla, 2011.

(23) SACMI IMOLA. Tecnología cerámica aplicada, Volume 1. Castellón, 2004.

(24) García-Portillo, C., Bastida, J., Pardo, P., Rodríguez-López, G., Lacruz, M. J., Vilar, M. L., Lazaro, A. Influencia de características microestructurales de caolinita en las propiedades de sus pastas de colaje. Bol. Soc. Esp. Ceram., 44, 3, 239-244, 2005.

(25) Davis B. L., Smith, D. Table of Experimental Reference Intensity Ratios. Powder Diffr., 3, 201-205, 1989.

(26) Davis B.L, D. Smith, D. Table of Experimental Reference Intensity Ratios. Powder Diffr., 4, 206-209, 1989.

(27) Zoellner, A. Zur Frage der Chemischem und Physikalischen Natur der Porzellans (Some Chemicals and physical properties of porcelains). Sprechsaal, 41,471-473, 1908.

(28) Escardino A., Amorós J.L., Enrique J. E. Estudio de pastas de gres para pavimentos. Bol. Soc. Esp. Ceram. Vidr., 20, 17-24, 1981

(29) Pardo, P., García-Portillo, C., De la Torre, F. J., Amigo, J. M., y Bastida, J. Caracterización microestructural de caolinita y gresificación de cuerpos cerámicos. Macla, 3, 149-150, 2005.

Recibido: 05/12/2009

Aceptado: 21/03/2012 